Proyecciones Journal of Mathematics

Vol. 32, No 3, pp. 281-288, September 2013.

Universidad Católica del Norte

Antofagasta - Chile

\title{
On the univalence of certain integral transform
}

\author{
Osvaldo Venegas \\ Universidad Católica de Temuco, Chile \\ Rodrigo Hernández \\ Universidad Adolfo Ibañez, Chile \\ Received : December 2012. Accepted : June 2013
}

\begin{abstract}
We study the univalence of $f_{\alpha}$, define as a integral of the power $\alpha$ of $f^{\prime}$, in terms of the values of $\alpha$ when $f$ belongs to certain subclasses of univalent functions.
\end{abstract}

Key Words : Univalent functions, Schwarzian derivatives, Integral transform. 


\section{Introduction}

Let $\mathcal{S}$ be the set of all holomorphic and univalent functions in the unit disk $=\{z:|z|<1\}$, normalized with $f(0)=0$ and $f^{\prime}(0)=1$. Let $\alpha$ be a complex number and defined the function $f_{\alpha}$ as

$$
f_{\alpha}(z)=\int_{0}^{z} f^{\prime}(w)^{\alpha} d w
$$

The power in the mapping $f_{\alpha}$ is defined via the branch of $\log f^{\prime}(w)$ for which $\log f^{\prime}(0)=0$.

It is trivial to check that given any $f \in \mathcal{S}$, if either $\alpha=1$ or $\alpha=0$, the corresponding function $f_{\alpha}$ belongs to $\mathcal{S}$. Nevertheless, the question of determinig the values of $\alpha$ for which the function $f_{\alpha}$ are in $\mathcal{S}$ when $f \in \mathcal{S}$ is not so easy.

Royster showed that, in general, $f_{\alpha}$ fails to be univalent for $|\alpha|>1 / 3$ and $\alpha \neq 1$. See $([9])$.

Pfaltzgraff in [7], proved that for any given univalent function $f$ in unit disk normalized as above, if $|\alpha| \leq 1 / 4$, then $f_{\alpha} \in \mathcal{S}$. It is an open problem to determine if (in general) $f_{\alpha}$ is univalent when $\frac{1}{4}<|\alpha| \leq \frac{1}{3}$.

If we let $f$ belong to some particular subclasses of functions in $\mathcal{S}$ (concretely, if $f$ is convex or close-to-convex), we can determine completely the values of $\alpha \in$ for which $f_{\alpha} \in \mathcal{S}$. [5, p. 152]].

The main purpose of this work is to determinate the values of $\alpha$ for which the function $f_{\alpha}$ is univalent when $f$ belongs to certain subclasses of $\mathcal{S}$ defined by certain conditions related to univalence criteria. Especifically, the Nehari and Pokornyi's criteria. See [6].

Recall that the Schwarzian derivative of a locally univalent function $f$ on a simply connected domain is defined by

$$
S f=\left(\frac{f^{\prime \prime}}{f^{\prime}}\right)^{\prime}-\frac{1}{2}\left(\frac{f^{\prime \prime}}{f^{\prime}}\right)^{2}
$$

It is well known that the Schwarzian derivative is invariant under Mobius transformations. It has turned out to be a very useful tool in the study of certain geometric properties of locally univalent functions. For instance, it can be used to get either necessary or sufficient conditions for the global univalence, or to obtain certain geometric conditions on the range of the 
function. Nehari ([6]) proved that if a locally univalent analytic function $f$ in the unit disk satisfies

$$
|S f(z)| \leq \frac{2}{\left(1-|z|^{2}\right)^{2}}, \quad|z|<1,
$$

then $f$ is univalent. The constant $C=2$ is sharp.

The Nehari class $\mathcal{N}$ is the set of functions $f$ defined in for which $f(0)=0, f^{\prime}(0)=1$, and such that (1.3) holds. This class was studied in [2]. Obviously $\mathcal{N} \subset \mathcal{S}$. Related to $\mathcal{N}$ is the class $\mathcal{N}$, defined as the family of functions in $\mathcal{N}$ such that $f^{\prime \prime}(0)=0$. Note that this normalization can be obtained by considering the function $f /\left(1-a_{2} f\right)$ where $2 a_{2}=f^{\prime \prime}(0)$, instead of $f$.

Other important univalence criteria, established by Pokornyi and proved by Nehari in [6], says that if $f$ is a locally univalent mapping defined in and

$$
|S f(z)| \leq \frac{4}{1-|z|^{2}}, \quad z \in
$$

then $f$ is univalent in the unit disk. We define $\mathcal{P}$, as the class of all analytic functions in the unit disk with the normalizations $f(0)=0, f^{\prime}(0)=1$, and $f^{\prime \prime}(0)=0$ and satisfying (1.4).

We will obtain sufficient conditions for the univalence of $f_{\alpha}$ when $f$ belongs to the classes $\mathcal{N}$, or $\mathcal{P}$,

\section{Results}

Let $f(z)=z+a_{2} z^{2}+\cdots$ be a function in $\mathcal{S}$. The well known coefficient estimate $\left|a_{2}\right| \leq 2$, due to Bieberbach $([4,5,8])$, implies that for any $|z|<1$,

$$
\left(1-|z|^{2}\right)\left|z \frac{f^{\prime \prime}}{f^{\prime}}(z)\right| \leq 6 \text {. }
$$

This inequality is satisfied by the function $f_{\alpha}$ as in (1.1) with $6|\alpha|$ instead of 6 . Using the Becker criterion for univalence (see [1], [4] or [8]) it follows that $f_{\alpha}$ is univalent if $|\alpha| \leq 1 / 6$.

If $f$ satisfies equations (1.3) or $(1.4)$ and $f^{\prime \prime}(0)=0$, then the constant 6 above can be improved, as the following lemma asserts. The proof of the lemma uses the same argument as in [3, Lemma 1]. 
Lemma 1. Let $f$ be a locally univalent mapping with $f^{\prime \prime}(0)=0$, satisfying $|S f(z)| \leq 2 p(|z|)$ for all $|z|<1$, where $p(x) \geq 0$ is a continuous function defined in $(-1,1)$. Then, for all $|z|<1$,

$$
\left|\frac{f^{\prime \prime}}{f^{\prime}}(z)\right| \leq w(|z|)
$$

where $w(x)$ is the solution of

$$
w^{\prime}-\frac{1}{2} w^{2}=2 p(x), w(0)=0 .
$$

Proof 1. Notice that $y=f^{\prime \prime} / f^{\prime}$ is a solution of

$$
y^{\prime}-\frac{1}{2} y^{2}=S f, \quad y(0)=0 .
$$

Consider the real function $u(x)=|y(x)|$ which is differentiable whenever $y(x) \neq 0$. Note that $u^{\prime}(x) \leq\left|y^{\prime}(x)\right|$. Define $h=u-w$ in the interval $(0,1)$.

Since $|S f(z)| \leq 2 p(|z|)$, we get that

$$
\begin{aligned}
h^{\prime}(x)=(u(x)-w(x))^{\prime} & \leq\left|y^{\prime}(x)\right|-w^{\prime}(x) \\
& \leq|S f(x)|-2 p(x)+\frac{1}{2}\left(|y(x)|^{2}-w^{2}(x)\right) \\
& \leq \frac{1}{2}(u(x)+w(x))(u(x)-w(x)) \\
& =\frac{1}{2}(u(x)+w(x)) h(x) .
\end{aligned}
$$

Using that $h(0)=0$, we obtain $h(x) \leq 0$ for all $x \in(0,1)$. Then $|y(x)| \leq$ $w(x)$ for all such $x$.

Along other rays from the origin we consider the function $u(t)=\left|y\left(t z_{0}\right)\right|$, $\left|z_{0}\right|=1$. The same argument shows now that $u(t) \leq w(|z|)$, and the lemma is proved.

Corollary 1. Let $f \in \mathcal{N}_{\text {, }}$, then

$$
\left|\frac{f^{\prime \prime}}{f^{\prime}}(z)\right| \leq \frac{2|z|}{\left(1-|z|^{2}\right)} .
$$


Proof 2. The function $w(x)=2 x /\left(1-x^{2}\right)$ defined for $x \in(0,1)$ is solution of

$$
w^{\prime}-\frac{1}{2} w^{2}=\frac{2}{\left(1-x^{2}\right)^{2}} .
$$

Use Lemma 1 to finish the proof.

Theorem 2. Let $f \in \mathcal{N}_{1}$. Then,

(i) if $|\alpha| \leq 1 / 2$, the function $f_{\alpha} \in \mathcal{S}$,

(ii) if $|\alpha|(|1-\alpha|+1) \leq 1$, then $f_{\alpha} \in \mathcal{N}_{\text {, }}$

Proof 3. (i) Since $f_{\alpha}^{\prime}(z)=\left(f^{\prime}(z)\right)^{\alpha}$, we obtain

$$
\frac{f_{\alpha}^{\prime \prime}}{f_{\alpha}^{\prime}}(z)=\alpha \frac{f^{\prime \prime}}{f^{\prime}}(z)
$$

By Corollary 2.2, we have that

$$
\left|\frac{f_{\alpha}^{\prime \prime}}{f_{\alpha}^{\prime}}(z)\right| \leq \frac{2|\alpha||z|}{1-|z|^{2}}
$$

But $|\alpha| \leq 1 / 2$, so that $f_{\alpha}$ satisfies the hypothesis of Becker's criterion of univalence which implies that $f_{\alpha}$ is univalent in .

(ii) Using (2.1) we get that $f_{\alpha}^{\prime \prime}(0)=\alpha f^{\prime \prime}(0)=0$. Straightforward computations show that

$$
S f_{\alpha}(z)=\alpha\left[S f(z)+\frac{1-\alpha}{2}\left(\frac{f^{\prime \prime}}{f^{\prime}}(z)\right)^{2}\right] .
$$

Therefore,

$$
\begin{aligned}
\left|S f_{\alpha}(z)\right| & \leq 2|\alpha|\left(\frac{1+|1-\alpha||z|^{2}}{\left(1-|z|^{2}\right)^{2}}\right) \\
& \leq \frac{2(|\alpha|(|1-\alpha|-1)+2|\alpha|)}{\left(1-|z|^{2}\right)^{2}} \leq 2 \frac{(|\alpha|(|1-\alpha|+1))}{\left(1-|z|^{2}\right)^{2}} .
\end{aligned}
$$


Since $|\alpha|(|1-\alpha|+1) \leq 1$, we conclude

$$
\left|S f_{\alpha}(z)\right| \leq \frac{2}{\left(1-|z|^{2}\right)^{2}} .
$$

Hence, $f_{\alpha} \in \mathcal{N}_{1}$.

Corollary 2. If $f \in \mathcal{P}$, then

$$
\left|\frac{f^{\prime \prime}}{f^{\prime}}(z)\right| \leq \frac{4|z|}{\left(1-|z|^{2}\right)}
$$

Proof 4. The real function $w(x)=4 x /\left(1-x^{2}\right)$ is a solution of

$$
w^{\prime}-\frac{1}{2} w^{2}=\frac{4}{\left(1-x^{2}\right)}, \quad w(0)=0 .
$$

Use Lemma 1, to obtain (2.3).

Remark 1. Using Lemma 1, we can show that given any $f \in \mathcal{P}_{\text {l, the }}$ corresponding function $f_{\alpha}$ is univalent if $|\alpha| \leq 1 / 4$. This was already proved in [7].

Theorem 3. Let $f$ be a locally univalent analytic mapping in $\mathcal{P}_{1}$. Then, if $4|\alpha(1-\alpha)| \leq 1$ and $2|1-\alpha| \geq 1$, then $f_{\alpha} \in \mathcal{N}_{1}$.

Proof 5. A straightforward computation shows

$$
\begin{aligned}
\left|S f_{\alpha}(z)\right| & \leq|\alpha|\left(|S f(z)|+\left|\frac{1-\alpha}{2}\right|\left|\frac{f^{\prime \prime}}{f^{\prime}}(z)\right|^{2}\right) \\
& \leq 4|\alpha| \frac{\left(1-|z|^{2}\right)+2|1-\alpha||z|^{2}}{\left(1-|z|^{2}\right)^{2}} \leq 4|\alpha| \frac{\left.1+(2|1-\alpha|-1)|z|^{2}\right)}{\left(1-|z|^{2}\right)^{2}} .
\end{aligned}
$$

Since $\alpha$ satisfies that $2|1-\alpha|-1 \geq 0$, the last expression is less than or equal to $2(4|\alpha(1-\alpha)|) /\left(1-|z|^{2}\right)^{2} \leq 2$. This implies that $f \in \mathcal{N}_{\text {I. }}$. 


\section{Acknowledgment}

We would like to thank Professor María José Martín for her helpful comments that improved the clarity of the exposition in this paper.

The first author was supported by DGIP 2009-2-1. The second author was supported in part by FONDECYT Grant \# 1110160.

\section{References}

[1] J. BECKER, Löwnersche Differentialgleichung und quasi-konform forttsetzbare schlichte Funktionen. J. Reine. Angew. Math., 225, pp. 23-43, (1972).

[2] M. Chuaqui, And B. Osgood, Sharp distortion theorems associated with the Schwarzian derivative. Jour. London Math. Soc., 2(48), pp. 289-298, (1993).

[3] M. Chunqui and B. Osgood, Ahlfors-Weill extensions of conformal mappings and critical points of the Poincaré metric. Comment. Math. Helv., 69, pp. 659-668, (1994).

[4] P.L. Duren, Univalent Functions, Springer-Verlag, New York, (1983).

[5] A.W. Goodman, Univalent Functions, Mariner Publishing Company, Florida, (1983).

[6] Z. NehaRI, The Schwarzian derivative and schlicht functions. Bull. Amer. Math. Soc., 55, pp. 545-551, (1949).

[7] J. A. Pfaltzgraff, Univalence of the integral of $f^{\prime}(z)^{\lambda}$. Bull. London Math. Soc., 7, pp. 254-256, (1975).

[8] Ch. Pommerenke, Univalent Functions, Vandenhoeck \& Ruprecht, Göttingen, (1975).

[9] W.C. Royster, On the univalence of a certain integral. Michigan Math. J., 12, pp. 385-387, (1965). 


\section{Osvaldo Venegas}

Departamento de Cs. Matemáticas y Físicas,

Facultad de Ingeniería,

Universidad Católica de Temuco,

Chile,

e-mail : ovenegas@uct.cl

and

\section{Rodrigo Hernández}

Facultad de Ingeniería y Ciencias, Universidad Adolfo Ibáñez,

Av. Padre Hurtado 750,

Viña del Mar,

Chile,

e-mail: rodrigo.hernandez@uai.cl 